\title{
Research Paper: Using Artificial Neural Network to Destroy the Process of Traffic Accident Victims in Yazd Province
}

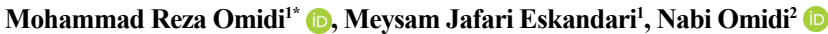 \\ 1. Department of Industrial Engineering, Payame Noor University of Tehran, Tehran, Iran. \\ 2. Department of Management, Payame Noor University of Tehran, Tehran, Iran.
}

\begin{tabular}{|l|l}
$\begin{array}{c}\text { Use yur devic to scan } \\
\text { and read the article online }\end{array}$ & $\begin{array}{l}\text { Citation: Omidi MR, Jafari Eskandari M, Omidi N. Using Artificial Neural Network to Destroy the Process of Traffic Acci- } \\
\text { dent Victims in Yazd Province. Health in Emergencies and Disasters Quarterly. 2021; 6(2):123-128 http://dx.doi.org/10.32598/ } \\
\text { hdq.6.2.222.10 }\end{array}$ \\
doi: $:$ http://dx.doi.org/10.32598/hdq.6.2.222.10
\end{tabular}

\section{(i) $\$$}

Article info:

Received: 21 Apr 2020

Accepted: 31 Nov 2020

Available Online: 01 Jan 2021

Keywords:

Crash, Trauma, Accidents

\section{ABSTRACT}

Background: Road accidents are among the most important causes of death and severe personal and financial injuries. Also, its profound social, cultural, and economic effects threaten human societies. This study aimed to estimate the trend of traffic accident victims in Yazd Province, Iran, to predict the number of traffic accident victims in this province.

Materials and Methods: Based on traffic casualty statistics referred to forensic medicine in Yazd Province within April 1989 and March 2017 referred to Forensic Medicine of Yazd Province and using an artificial neural network to predict the number of injured for 12 months ending in 2020 has been paid. The neural network used in this study had 12 inputs, one output, and 5 hidden layers. The network predicts the relationship between data after training and learning. The network is considered the MSE benchmark.

Results: The number of injured in traffic accidents in Yazd Province in 2020 was equal to 7052 people, with the highest number in December with 832 people and the lowest in June with 414 people. The exact method of use was equal to 92 cases.

Conclusion: The trend of traffic accident casualties in Yazd Province in 2020 will be declining. For future research, the exact method designed in this study can be examined with other methods for the best response level.

\section{Introduction}

oad accidents are one of the critical phenomena observed following the $\mathbf{R}$ motorization of communities and the increasing use of motor vehicles. Road accidents lead to the deaths or injuries of many people every year. Financial losses and psychological damages are some of the consequences of this phenomenon [1].
Nowadays, in all societies, either developed or developing, there is a great emphasis on providing accurate and correct statistics because statistical forecasting results can be considered a powerful tool for making appropriate decisions and correctly allocating resources [2]. Road traffic accidents are a significant public health problem worldwide, so that traffic accidents are one of the leading causes of death, disability, and hospitalization leading to significant social and economic consequences [3]. It is anticipated that in the coming years,

* Corresponding Author:

Mohammad Reza Omidi, PhD.

Address: Department of Industrial Engineering, Payame Noor University of Tehran, Tehran, Iran

E-mail: mromid91@yahoo.com 
traffic accidents are the second leading cause of death in high-income and middle-income countries [4]. Studies on traffic accidents show that by 2025, the number of traffic accidents will increase to more than 2.5 million [5], with developing countries more affected. According to the World Health Organization, without preventive measures to reduce accidents, the share of traffic deaths will increase to more than $67 \%$ [6]. Today road safety is one of the essential concerns worldwide. Every year, a lot of money is spent on reducing accidents and deaths caused by these accidents in different countries [7]. Increasing the number of vehicles and drivers' lack of attention to road safety regulations have been the most critical factors affecting the number and severity of traffic accidents in Iran [8]. Besides reducing safety, accidents cause considerable costs to society. Financial and human losses, traffic delays, and traffic slowing are some of the effects of accidents on society, reducing national capital.

Accident as a destructive phenomenon is one of the most critical factors in controlling transportation infrastructure. Using strategies to reduce accidents and road casualties requires analyzing accidents using prediction models and the impact of various parameters on their occurrence and efforts to improve traffic safety [9]. Predictive models have many applications in road safety analysis and are widely used in identifying factors affecting road safety, identifying and prioritizing accident-prone road segments, evaluating the safety of the transportation system, and projects resulting from macro-transportation plans and risk index determination are used to determine the safest route in the dynamic system of roads [10]. Researchers use various methods to predict accidents, such as time series technique, gray methods, Holt-Winters forecasting procedure, multivariate analysis, Bayesian forecasting approach, artificial neural network methods, and fuzzy logic-based methods. All these methods require the collection of sufficient data to compensate for their limitations. Predicting the number of accidents is significant and vital not only for governments but also for companies and organizations [11]. Considering the importance of predicting traffic accidents to make strategic decisions on road safety, we aimed to estimate the trend of traffic accident casualties in Yazd Province to estimate the number of traffic accident casualties in this province.

\section{Materials and Methods}

The present study is a comparative descriptive and analytical study to predict the future trend of accidents using the available information. This study aimed to predict the number of injured through 12 months of 2020 and measure the accuracy of the artificial neural network method in predicting traffic accidents. The data used in this study include the number of injured and deaths in traffic accidents according to provinces of Iran between 1989 and 2019, which were obtained from the forensic information database and Rahvar Naja Police Planning Organization. The forecasting method of this study was univariate; thus, the data are only related to the injured in traffic accidents in Yazd Province, Iran. MATLAB software was used for neural network prediction. In this study, an artificial neural network with 12 inputs (previous real values) and optimal hidden layers, and an output layer were used. The data were divided into experimental and educational categories using artificial neural networks and the network forecasted for the next 12 months of 2020 after training. The network repetitions were stopped after 50000 repetitions and achieving high accuracy in output. The mean absolute percentage error (MAPE) was used to measure accuracy. In the MLP network, neurons are organized in successive layers. The first layer is called the input layer, which contains neurons that receive input from the real world. The last layer is the output layer that provides the system output. The layers between these two layers are hidden layers, which can be more than one. Hidden layers indicate the network's ability to extract useful information from input data and return it to the target output.

In this study, the connections between input and output neurons are indirect, indicating that the input neurons are not directly connected to outputs but connected through hidden layer neurons, and also neurons have no reverse connection. The weighted input signals of each neuron in the first layer provide their output through the linear combination of the input signal weights with the activation function. The output as input is transmitted directly to the hidden layer, and this is repeated sequentially until it reaches the last layer and the output of the output layer is created. Figure 4 displays the basic structure of the MLP network with a hidden layer. MLP acts as a mapping function for the input space to the output space. However, the input and output dimensions are different. The number of input neurons does not have to be equal to the output. The network shown in Figure 1 has $\mathrm{N}$ inputs and L outputs. The relationship between inputs and outputs is determined by synaptic weights and biases. In most short-term forecasting applications, a three-layer MLP network is used.

Sigmoid functions are generally preferred for hidden layers. For output units, the output function can be both linear and nonlinear, which is selected based on the distribution of output variables. For example, a sigmoid 
Table 1. Neural network accuracy in predicting injuries in Yazd Province

\begin{tabular}{|c|c|c|}
\hline \multirow{2}{*}{ Neural Network } & \multicolumn{2}{|c|}{ Yazd Province } \\
\hline & Experimental Data & Training Data \\
\hline Number of data & 60 & 296 \\
\hline Number of correct predictions (good) & $53(88 \%)$ & $239(81 \%)$ \\
\hline Number of wrong predictions (bad) & $7(12 \%)$ & $57(19 \%)$ \\
\hline The mean absolute percentage error & 0.081 & \\
\hline
\end{tabular}

function can be used for an output with a continuous and finite value. When the output borders are infinite and unlimited activation function, such as a linear function, may be a better choice. A direct-fed MLP with at least one hidden layer can estimate any input-output relationship with reasonable accuracy. This kind of neural network is sometimes called a general estimator.

The mathematical algorithm used to update network parameters is known as the law of learning. There is no standard rule for different types of networks, and different rules are selected depending on the nature of the issue. ANN learning can generally be divided into two categories: supervised and unsupervised.
In supervised learning, also called teacher-learning, specific patterns are provided to the network, and the network adjusts its parameters according to those patterns. But if new information is provided to the network, the network selects the most appropriate pattern that fits the input information and gives it to the output. In supervised learning, typically, gradient descent is used to find an optimal path with the lowest level of error [12].

\section{Results}

Figure 1 displayed the time series of traffic injured in Yazd Province between April 1989 and March 2019. According to the months of the year, the number of injured

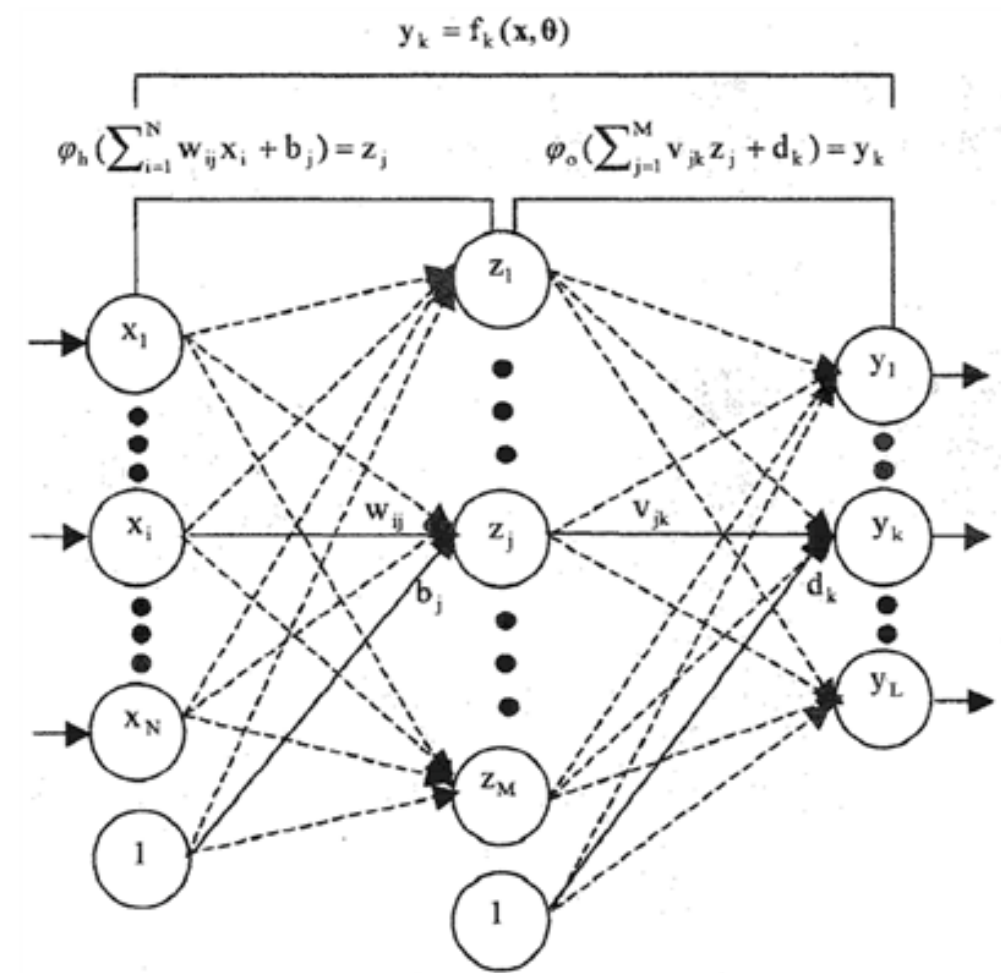

Figure 1. A 3-layer MLP network 


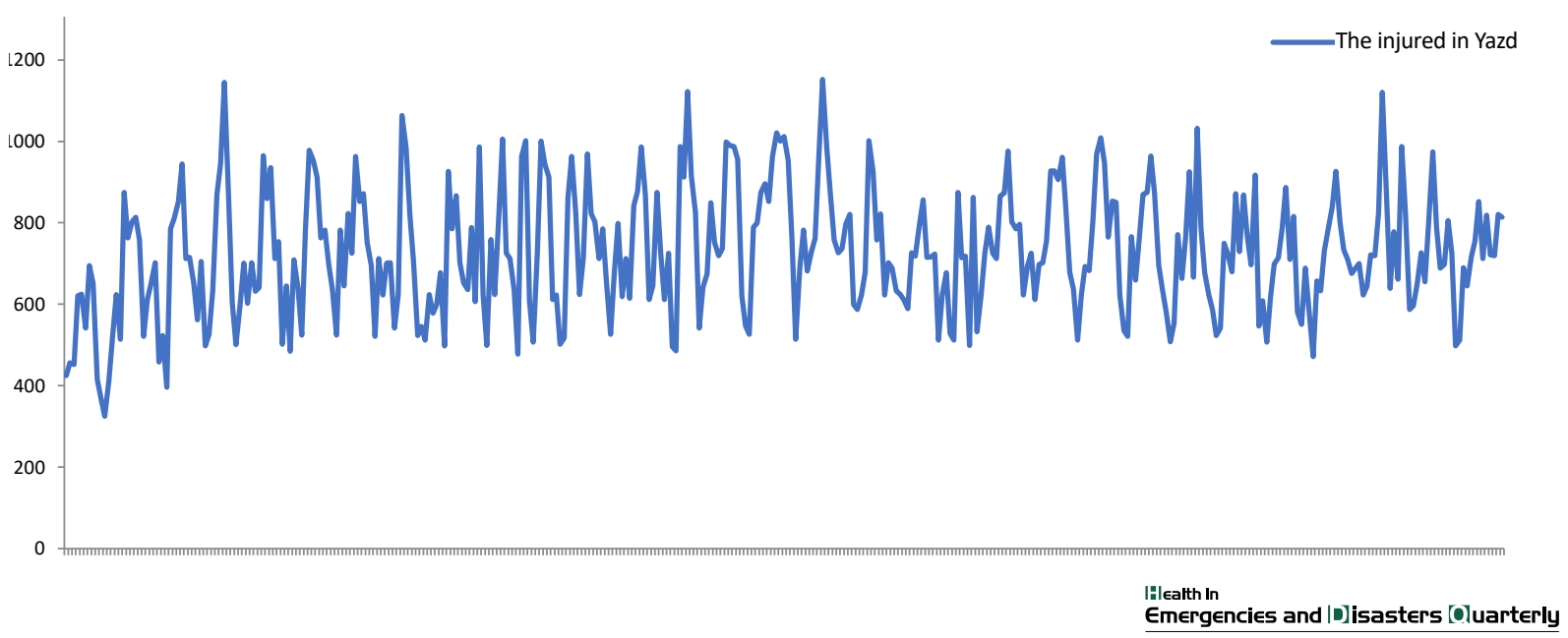

Figure 2. Time series chart of the number of injured referred to forensic medicine in Yazd Province

in Yazd Province in previous years has had a relatively constant trend with a decreasing tendency.

The neural network is designed to have 12 inputs and divided the data into 296 training data and 60 experimental data. The number of hidden layers for the network was obtained by five layers according to measuring the accuracy for different hidden layers. Table 1 presents the accuracy of the artificial neural network method.

The trained artificial neural network is then used to predict the 12-month one-step-ahead forecasting (Table 2 ). According to the forecast made by the artificial neural network, the number of injured in traffic accidents in
Yazd Province in 2020 is 7052 people, with the highest number in December with 832 people and the lowest in June with 414 people.

Based on the repetition of accidents in Yazd Province, the neural network predicted the rate of accidents. Also, Figure 6 shows the rate of errors of each repetition to achieve the desired prediction.

\section{Discussion}

Every year, many people in Iran lose their lives due to traffic accidents and trauma or face various injuries that cause social and economic costs for both individuals and

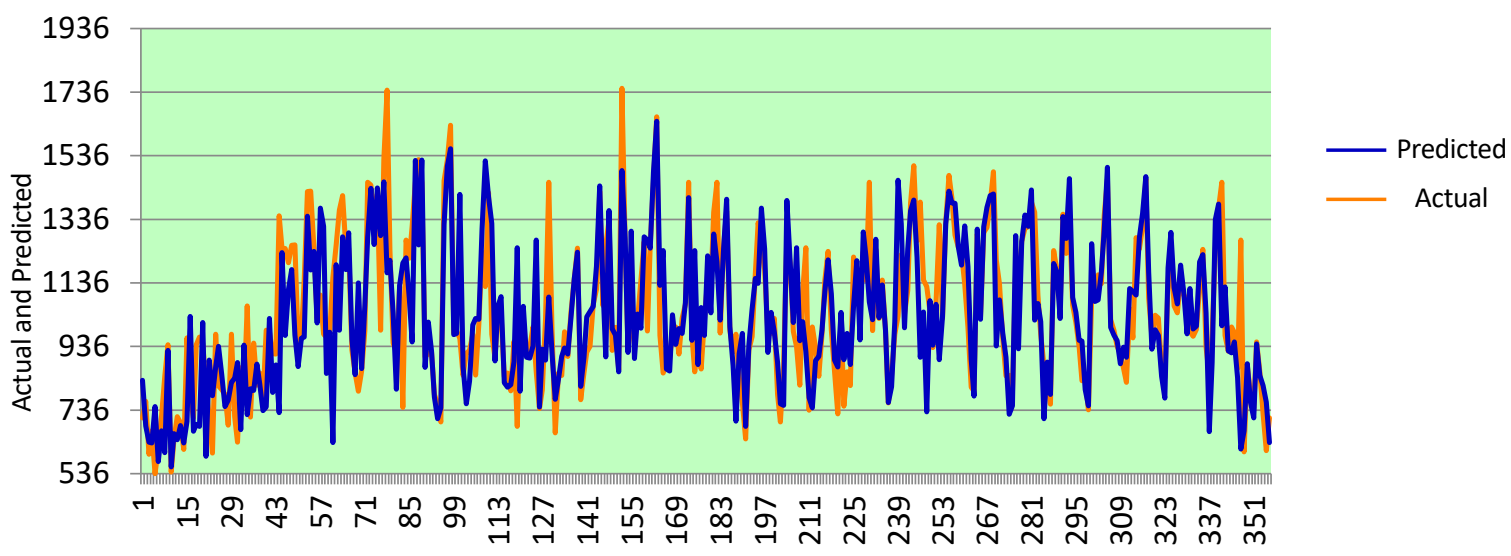

Figure 3. Actual values versus predicted values

Hlealth in
Emergencies and |Disasters [Oluarterly

Table 2. The one-step-ahead forecasted values for provinces using artificial neural network

\begin{tabular}{|c|c|c|c|c|c|c|c|c|c|c|c|c|}
\hline City Step & First & Second & Third & Fourth & Fifth & Sixth & Seventh & Eighth & Ninth & Tenth & Eleventh & MAPE \\
\hline Yazd & 511 & 587 & 649 & 414 & 461 & 633 & 478 & 522 & 832 & 749 & 667 & 549 \\
\hline
\end{tabular}




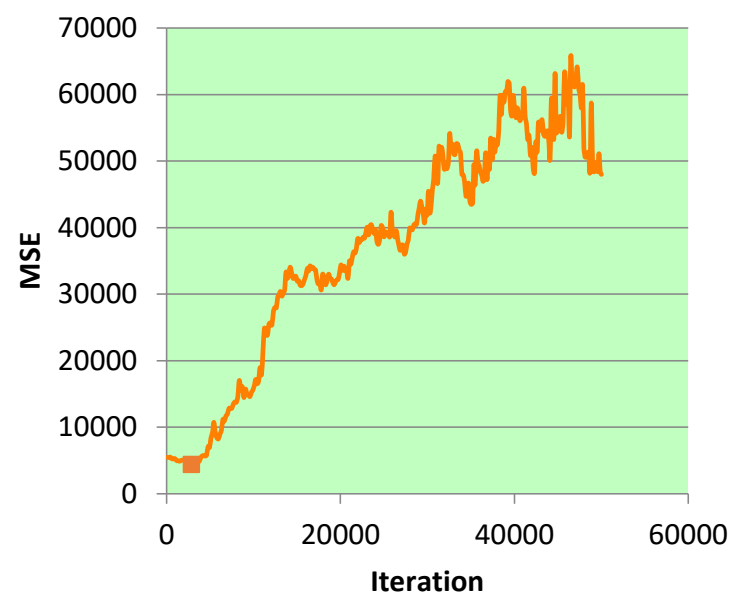

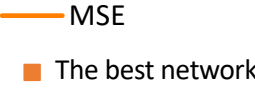

The best network

Figure 6. Mechanism of repetitions to achieve optimal accuracy

Health in

Emergencies and |D]isasters [O]uarterly society. In this study, we tried to measure the accuracy of the artificial neural network and predict the number of injured in traffic accidents in Yazd province using advanced methods of artificial neural network prediction. The accuracy of prediction methods was measured according to the MAPE. The results of the accuracy of forecasting methods showed that the neural network forecasting method has good accuracy in predicting traffic accidents. These results are consistent with the results of studies by Bahadori Monfared et al. (2013), who examined the rate of traffic accidents in Iran [13] and Omidi and Omidi (2018). Omidi et al. (2019) presented an approach to increase the accuracy of accident prediction methods, and contrary to the results of the present research, Box-Jenkins method was more accurate than other prediction methods, including artificial neural networks [7]. Asgari et al. (2019) also introduced the ARIMA time series method as an accurate method for predicting traffic accidents in Iran [5].

Comparing the accuracy of traffic accident forecasting methods with the accuracy of the method used in this research shows that predictive methods based on the nature of the data show different accuracy, and in addition to the capacity of the predictive method, the nature of the data also affect the accuracy of the forecasting methods. The predicted values for the next year in Yazd Province showed that the number of injured due to accidents in this province decreased, consistent with the study by Bahadori Monfared et al. (2013), who reported a decreasing trend of traffic accidents in Iran [13]. Omidi et al. also found a decrease in the rate of traffic accidents in Zanjan Province [14, 15]. In Asgari et al. (2019) study, the trend of traffic accident casualties based on econometric models in Iran was declining [5]. According to Chukwutoo et al., the number of accidents in Nigeria will increase in the future [16]. The result of the present study and its comparison with the studies conducted on the trend of traffic accident victims showed that the trend of traffic accidents in Iran is a downward trend.

Given that predicting the picture of the future helps managers to make tactical and strategic decisions, it is suggested that experts use the method presented in this research to predict and make decisions. It is suggested that in future research, different prediction methods be used to measure the rate of accidents. The decrease in the number of injured in Yazd Province shows the effectiveness of the traffic police in this province.

\section{Ethical Considerations}

\section{Compliance with ethical guidelines}

All ethical principles are considered in this article.

\section{Funding}

This research did not receive any grant from funding agencies in the public, commercial, or non-profit sectors.

\section{Authors' contributions}

All authors equally contributed to preparing this article.

\section{Conflict of interest}

The authors declared no conflict of interest. 


\section{References}

[1] Kurakina E, Evtiukov S, Rajczyk J. Forecasting of road accident in the DVRE system. Transportation Research Procedia 2018; 36:380-5. [DOI:10.1016/j.trpro.2018.12.111]

[2] Fountas G, Anastasopoulos PC, Mannering FL. Analysis of vehicle accident-injury severities: A comparison of segmentversus accident-based latent class ordered probit models with class-probability functions. Analytic Methods in Accident Research. 2018; 18:15-32. [DOI:10.1016/j.amar.2018.03.003]

[3] Omidi N, Omidi MR. Estimating accident-related traumatic injury rate by future studies models in Semnan province, Iran. Health in Emergencies and Disasters Quarterly. 2018; 3(4):191-8. [DOI:10.32598/hdq.3.4.191]

[4] Grant E, Salmon PM, Stevens NJ, Goode N, Read GJ. Back to the future: What do accident causation models tell us about accident prediction? Safety Science. 2018; 104:99-109. [DOI:10.1016/j.ssci.2017.12.018]

[5] Asgari H, Omidi MR, Omidi N. [Use of econometric techniques to estimate the traumatic trend of road accidents (Persian)]. Journal of Safety Promotion and Injury Prevention. 2019; 6(4):173-83. [DOI:10.22037/meipm.v6i4.25491]

[6] Fountas G, Anastasopoulos PC. Analysis of accident injuryseverity outcomes: The zero-inflated hierarchical ordered probit model with correlated disturbances. Analytic Methods in Accident Research. 2018; 20:30-45. [DOI:10.1016/j. amar.2018.09.002]

[7] Omidi MR, Jafari Eskandari M, Raissi S, Shojaei AA. Providing an appropriate prediction model for traffic accidents: A case study on accidents in Golestan, Mazandaran, Guilan, and Ardebil provinces. Health in Emergencies and Disasters Quarterly. 2019; 4(3):165-72. [DOI:10.32598/hdq.4.3.171]

[8] Tabrizi R, Akbari M, Lankarani KB, Heydari ST, Masoudi A, Shams AH, et al. Relationship between religion and school students' road behavior in southern Iran. Chinese Journal of Traumatology. 2017; 20(5):264-9. [DOI:10.1016/j.cjtee.2016.12.001]

[9] Mannering F. Temporal instability and the analysis of highway accident data. Analytic Methods in Accident Research 2018; 17:1-13. [DOI:10.1016/j.amar.2017.10.002]

[10] Mehdizadeh M, Shariat-Mohaymany A, Nordfjaern T. Accident involvement among Iranian lorry drivers: Direct and indirect effects of background variables and aberrant driving behaviour. Transportation Research Part F: Traffic Psychology and Behaviour. 2018; 58:39-55. [DOI:10.1016/j.trf.2018.05.029]

[11] Mohammadzadeh Moghaddam A, Tabibi Z, Sadeghi A, Ayati E, Ghotbi Ravandi A. Screening out accident-prone Iranian drivers: Are their at-fault accidents related to driving behavior? Transportation Research Part F: Traffic Psychology and Behaviour. 2017; 46(Part B):451-61. [DOI:10.1016/j.trf.2016.09.027]

[12] Naderi M, Khamehchi E, Karimi B. Novel statistical forecasting models for crude oil price, gas price, and interest rate based on meta-heuristic bat algorithm. Journal of Petroleum Science and Engineering. 2019; 172:13-22. [DOI:10.1016/j.petrol.2018.09.031]

[13] Bahadori Monfared A, Soori H, Mehrabi Y, Rahmati Roudsar M, Esmaili A, Salehi M, et al. [A model for prediction of on the rate of mortality due to road traffic accidents in Iran (Persian)]. Research-in-Medicine. 2013; 36(5):7-11. http://pejouhesh.sbmu.ac.ir/article-1-1096-en.htm
[14] Omidi MR, Omidi N, Mahmoudian Azar Sharabiani A Evaluation of decision-making units in reducing traffic accidents using data envelopment analysis. Health in Emergencies and Disasters. 2020; 5(2):105-14. [DOI:10.32598/hdq.5.2.222.7]

[15] Omidi N, Asgari H, Omidi MR, Jafari Eskandari M. [The study of traffic accidents in Zanjan Province between March 2009 and February 2016 and comparing the mathematical method to predict traffic injuries referred to the Forensic Medicine between 2017 and 2020 (Persian)]. Medical Sciences Journal of Islamic Azad University , Tehran Medical Branch. 2017 27(3):201-8. http:/ / tmuj.iautmu.ac.ir/article-1-1299-en.html

[16] Ihueze CC, Onwurah UO. Road traffic accidents prediction modelling: An analysis of Anambra State, Nigeria. Accident Analysis \& Prevention. 2018; 112:21-9. [DOI:10.1016/j. aap.2017.12.016] 